\title{
Path Integral Quantization of the Aharonov-Bohm-Coulomb System in Momentum Space
}

\author{
De-Hone Lin $^{1}$ \\ Institute of Electro-Physics, National Chiao Tung University, Hsinchu 30043, Taiwan
}

Received November 13, 2000; revised February 5, 2001

\begin{abstract}
The Coulomb system with a charge moving in the fields of Ahanorov and Bohm is quantized via path integral in momentum space. Due to the dynamics of the system in momentum space being in curve space, our result not only gives the Green function of this interesting system in momentum space but provides the second example to answer an open problem of quantum dynamics in curved spaces posed by DeWitt in 1957: We find that the physical Hamiltonian in curved spaces does not contain the Riemannian scalar curvature $R$. @ 2001 Academic Press
\end{abstract}

\section{INTRODUCTION}

Half a century ago, Feynman proposed the method of the path integral to describe quantum dynamics $[1,2]$. It provides us with a global approach for studying quantum dynamics via fluctuating paths. Feynman's method has been successfully applied to diverse areas of physics $[3,4]$. Nevertheless, so far almost all the exact results for the dynamic problems of particles moving in the external potentials given by the path integral are obtained in position space $[5,6]$. In this paper, we calculate the path integral of the Aharonov-Bohm-Coulomb (A-B-C) system in momentum space. Our procedures can serve a further example for treating quantum dynamics in momentum space by path integrals. Because the dynamics of the system in momentum space is in curved space, it may give an answer to an old question in quantum dynamics in curved spaces proposed by DeWitt in 1957: The Hamiltonian in curved spaces should not contain an additional term of the Riemannian scalar curvature $R$ [7]. Otherwise, the level spacing of the energy spectra will be changed. The phenomenon was first observed by Kleinert in Ref. [8] who pioneered the treatment of the Coulomb system in momentum space. Here we provide the second example to confirm his result.

\section{PATH INTEGRAL QUANTIZATION OF THE A-B-C SYSTEM IN MOMENTUM SPACE}

With the space-time transformation, a stable path integral representation for the quantum Green function of a charge particle moving in the external electromagnetic fields from $\mathrm{x}_{a}$ to $\mathrm{x}_{b}$ is given by $[8,10,11]$

$$
G\left(\mathrm{x}_{b}, \mathrm{x}_{a} ; E\right)=\int_{0}^{\infty} d S \int \mathcal{D} f(\lambda) \Phi[f(\lambda)] \int \mathcal{D}^{3} x(\lambda) \exp \left\{-\mathcal{A}_{E}[\mathrm{x}, \dot{\mathrm{x}}]\right\}
$$

\footnotetext{
${ }^{1}$ E-mail: dhlin@cc.nctu.edu.tw.
} 
with the classical action

$$
\mathcal{A}_{E}[\mathrm{x}, \dot{\mathrm{x}}]=\int_{\lambda_{a}}^{\lambda_{b}} d \lambda\left[\frac{\dot{\mathrm{x}}^{2}(\lambda)}{2 f(\lambda)}-i e \mathbf{A}(\mathrm{x}) \cdot \dot{\mathrm{x}}(\lambda)+f(\lambda)(V(\mathrm{x})-E)\right],
$$

where $S$ is defined as

$$
S=\int_{\lambda_{a}}^{\lambda_{b}} d \lambda f(\lambda)
$$

in which $f(\lambda)$ is an arbitrary dimensionless fluctuating scale variable, and $\Phi[f(\lambda)]$ is some convenient gauge-fixing functional. The only condition on $\Phi[f(\lambda)]$ is that

$$
\int \mathcal{D} f(\lambda) \Phi[f(\lambda)]=1
$$

In Eq. (2.1), natural units with $\hbar=c=m=1$ are used. We see that if $\Phi[f(\lambda)]$ is taken as the delta functional $\delta[f-1]$, the representation reduces to Feynman's original path integral of a relativistic particle. The path integral representation arises from the continuous limit of the $\lambda$-sliced expression

$$
G\left(\mathrm{x}_{b}, \mathrm{x}_{a} ; E\right) \approx \int_{0}^{\infty} d S \prod_{n=0}^{N}\left[\int d f_{n} \Phi\left(f_{n}\right)\right] \frac{1}{\left(2 \pi \epsilon_{b} f_{b}\right)^{3 / 2}} \prod_{n=1}^{N}\left[\int_{-\infty}^{\infty} \frac{d^{3} x_{n}}{\left(2 \pi \epsilon_{n} f_{n}\right)^{3 / 2}}\right] \exp \left\{-\mathcal{A}_{E}^{N}\right\}
$$

with the $\lambda$-sliced action

$$
\mathcal{A}_{E}^{N}=\sum_{n=0}^{N}\left[\frac{\left(\mathrm{x}_{n}-\mathrm{x}_{n-1}\right)^{2}}{2 \epsilon_{n} f_{n}}-i e \mathbf{A}\left(\mathrm{x}_{n}\right) \cdot\left(\mathrm{x}_{n}-\mathrm{x}_{n-1}\right)+\epsilon_{n} f_{n}\left(V\left(\mathrm{x}_{n}\right)-E\right)\right],
$$

where $\epsilon_{n}=\lambda_{n}-\lambda_{n-1}, \lambda_{b}=\lambda_{N}, \lambda_{a}=\lambda_{0}, \mathrm{x}_{a}=\mathrm{x}\left(\lambda_{0}\right)$, and $\mathrm{x}_{b}=\mathrm{x}\left(\lambda_{N}\right)$. In the A-B-C potential problem that we consider, the vector and scalar potentials are defined as

$$
\mathbf{A}(\mathrm{x})=2 g \frac{-y \hat{e}_{x}+x \hat{e}_{y}}{x^{2}+y^{2}}, \quad V(r)=-\frac{\alpha}{r},
$$

where $\hat{e}_{x, y}$ stand for the unit vector along the $x, y$ axis. Let's first analyze the influence of A-B effect on the $G\left(\mathrm{x}_{b}, \mathrm{x}_{a} ; E\right)$. Introducing the azimuthal angle around the $\mathrm{A}-\mathrm{B}$ tube

$$
\varphi(\mathrm{x})=\arctan (y / x)
$$

the components of the vector potential can be expressed as

$$
A_{i}=2 g \partial_{i} \varphi(\mathrm{x})
$$

The associated magnetic field lines are confined to an infinitely thin tube along the z-axis,

$$
B_{3}=2 g \epsilon_{3 i j} \partial_{i} \partial_{j} \varphi(\mathrm{x})=4 \pi g \delta\left(\mathrm{x}_{\perp}\right),
$$


where $\mathrm{x}_{\perp}$ stands for the transverse vector $\mathrm{x}_{\perp}=(x, y)$. Note that the derivatives in front of $\varphi(\mathrm{x})$ commute everywhere, except at the origin where Stokes' theorem yields

$$
\int d^{2} x\left(\partial_{x} \partial_{y}-\partial_{y} \partial_{x}\right) \varphi(\mathrm{x})=\oint d \varphi=2 \pi
$$

The magnetic flux through the tube is defined by the integral

$$
\Omega=\int d^{2} x B_{3}
$$

This shows that the coupling constant $g$ is related to the magnetic flux by

$$
g=\frac{\Omega}{4 \pi} .
$$

Inserting $A_{i}=2 g \partial_{i} \varphi(\mathrm{x})$ into the action of Eq. (2.2), the magnetic interaction takes the form

$$
\mathcal{A}_{\text {mag }}=i \mu_{0} \int_{0}^{S} d \lambda \dot{\varphi}(\lambda)
$$

where $\varphi(\lambda)=\varphi(\mathrm{x}(\lambda)), \dot{\varphi}=d \varphi / d \lambda$, and $\mu_{0}$ is the dimensionless number

$$
\mu_{0}=-2 e g
$$

The minus sign is a matter of convention. Since the particle orbits are present at all times, their worldlines in space-time can be considered as being closed at infinity, and the integral

$$
k=\frac{1}{2 \pi} \int_{0}^{S} d \lambda \dot{\varphi}(\lambda)
$$

is the topological invariant with integer values of the winding number $k$. The magnetic interaction is therefore purely topological; its value is given by

$$
e^{-\mathcal{A}_{\mathrm{mag}}}=e^{i e \int_{\lambda_{a}}^{\lambda_{b}} d \lambda \mathbf{A}(\mathrm{x}) \cdot \dot{\mathrm{x}}(\lambda)}=e^{-i \mu_{0} 2 k \pi} .
$$

Due to the nature of topological interaction, the influence of the A-B effect may be considered after the dynamics of the Coulomb system in the momentum space is carried out. To perform the path integral of the Coulomb system in the momentum space, we note that the representation of the path integral in Eq. (2.1) has the phase space version

$$
G\left(\mathbf{p}_{b}, \mathbf{p}_{a} ; E\right)=\int_{0}^{\infty} d S \int \mathcal{D} f \Phi[f] \int \frac{\mathcal{D}^{3} p}{2 \pi} \int \mathcal{D}^{3} x \exp \{-\mathcal{A}[\mathbf{p}, \mathbf{x}, f]\}
$$

where the action

$$
\mathcal{A}[\mathbf{p}, \mathrm{x}, f]=\int_{0}^{S} d \lambda[i \dot{\mathbf{p}} \cdot \mathrm{x}+f(\mathcal{H}(\mathbf{p}, \mathrm{x})-E)]
$$


with $\mathcal{H}$ is the classical Hamiltonian. For the Coulomb system under consideration, $\mathcal{H}=\mathbf{p}^{2} / 2-\alpha / r$. In order to obtain a stable path integral in this case, the gauge fixing functional $\Phi[f]$ is chosen as [8]

$$
\Phi[f] \approx \prod_{n=0}^{N}\left[\frac{1}{r_{n}}\right] \exp \left\{-\sum_{n=0}^{N} \frac{\epsilon_{n}}{2 r_{n}^{2}}\left[f_{n}-\mathrm{x}_{n}^{2}\left(\frac{\mathbf{p}_{n}^{2}}{2}-E\right)\right]^{2}\right\} .
$$

With the functional measure

$$
\int \mathcal{D} f \approx \prod_{n=0}^{N}\left[\int_{-\infty}^{\infty} \frac{d f_{n}}{\sqrt{2 \pi / \epsilon_{n}}}\right]
$$

the gauge condition $\int \mathcal{D} f \Phi[f]=1$ is automatically satisfied. Combining Eq. (2.20), we have the action in the path integral

$$
\mathcal{A}[\mathbf{p}, \mathrm{x}, f]=\int_{0}^{S} d \lambda\left[i \dot{\mathbf{p}} \cdot \mathrm{x}+\frac{1}{2} \mathrm{x}^{2}\left(\frac{\mathbf{p}^{2}}{2}-E\right)^{2}+\frac{f^{2}}{2 r^{2}}-\frac{f \alpha}{r}\right] .
$$

We see that the path integrals over $f$ and $\mathrm{x}$ in this equation are Gaussian and can be performed. The time-sliced path integral in $f$ gives us a factor

$$
\exp \left\{\sum_{n=0}^{N} \frac{\epsilon_{n} \alpha^{2}}{2}\right\}
$$

The integrand of the time-sliced path integral associated with $\mathrm{x}$ reads now

$$
\prod_{n=0}^{N} \frac{2^{3}\left(2 \pi / \epsilon_{n}\right)^{3 / 2}}{\left(\mathbf{p}_{n}^{2}+\kappa^{2}\right)^{3}} \exp \left\{-\frac{1}{2} \sum_{n=0}^{N} \frac{\left(2 \triangle \mathbf{p}_{n}\right)^{2}}{\epsilon_{n}\left(\mathbf{p}_{n}^{2}+\kappa^{2}\right)^{2}}\right\},
$$

where we have defined $\kappa^{2} \equiv-2 E$. If the measure of the path integral is defined as in Dewitt's paper [7], the path integral becomes the form

$$
G\left(\mathbf{p}_{b}, \mathbf{p}_{a} ; E\right) \approx \int_{0}^{\infty} d S \frac{2^{3}(2 \pi)^{3}}{\left(2 \pi \epsilon_{a}\right)^{3 / 2}\left(\mathbf{p}_{a}^{2}+\kappa^{2}\right)^{3}} \prod_{n=1}^{N}\left[\int_{-\infty}^{\infty} \frac{2^{3} d^{3} p_{n}}{\left(2 \pi \epsilon_{n}\right)^{3 / 2}\left(\mathbf{p}_{n}^{2}+\kappa^{2}\right)^{3}}\right] \exp \left\{-\mathcal{A}_{E}^{N}\right\} .
$$

The sliced action reads

$$
\mathcal{A}_{E}^{N}=\sum_{n=0}^{N}\left[\frac{1}{2} \frac{\left(2 \triangle \mathbf{p}_{n}\right)^{2}}{\epsilon_{n}\left(\mathbf{p}_{n}^{2}+\kappa^{2}\right)^{2}}-\frac{\epsilon_{n} \alpha^{2}}{2}\right]
$$

Here we perform the stereographic projection onto a unit sphere in the four dimensions

$$
\mathbf{z}=\frac{2 \kappa \mathbf{p}}{\mathbf{p}^{2}+\kappa^{2}}, \quad z=\frac{\mathbf{p}^{2}-\kappa^{2}}{\mathbf{p}^{2}+\kappa^{2}}, \quad \mathbf{z}^{2}+z^{2}=1 .
$$


Then, Eq. (2.26) becomes

$$
\mathcal{A}_{E}^{N}[\hat{\mathbf{z}}]=\sum_{n=0}^{N}\left[\frac{1}{2} \frac{\left(\triangle \hat{\mathbf{z}}_{n}\right)^{2}}{\epsilon_{n} \kappa^{2}}-\frac{\epsilon_{n} \alpha^{2}}{2}\right]
$$

and the measure in Eq. (2.26),

$$
\int_{0}^{\infty} d S \frac{(2 \pi)^{3}}{\left(2 \pi \kappa^{2} \epsilon_{a}\right)^{3 / 2}} \prod_{n=1}^{N}\left[\int_{-\infty}^{\infty} \frac{d^{4} \hat{\mathbf{z}}_{n}}{\left(2 \pi \kappa^{2} \epsilon_{n}\right)^{3 / 2}}\right]
$$

where $\hat{\mathbf{z}}=(\mathbf{z}, z)$ is the four-dimensional unit vector. By defining a pseudomass $\mu=1 / \kappa^{2}$, we find the momentum path integral

$$
G\left(\mathbf{p}_{b}, \mathbf{p}_{a} ; E\right)=(2 \pi)^{3} \int_{0}^{\infty} d S K\left(\mathbf{p}_{b}, \mathbf{p}_{a} ; S\right)
$$

with the time sliced pseudo-propagator

$$
K\left(\mathbf{p}_{b}, \mathbf{p}_{a} ; S\right) \approx \frac{1}{\left(2 \pi \epsilon_{a} / \mu\right)^{3 / 2}} \prod_{n=1}^{N}\left[\int_{-\infty}^{\infty} \frac{d^{4} \hat{\mathbf{z}}_{n}}{\left(2 \pi \epsilon_{n} / \mu\right)^{3 / 2}}\right] \exp \left\{-\mathcal{A}_{E}^{N}[\hat{\mathbf{z}}]\right\}
$$

This would be the path integral of DeWitt. It should not lead to the correct spectrum for this problem. However, it was shown by Kleinert [5] that the measure of path integrals in curved space is not simply the product of invariant integrals $\prod_{n} \int d q_{n} \sqrt{g\left(q_{n}\right)}$. By performing a non-holonomic mapping from flat to curved space, he found that the measure receives a contribution explicitly,

$$
\mathcal{A}_{\text {eff }}=-\int_{0}^{S} d \lambda \frac{R}{6 \mu}
$$

in which $R$ is the Riemannian scalar curvature. For a sphere of radius $r$ in $D$ dimensional space, $R$ is given by $(D-1)(D-2) / r^{2}$, so that the measure correction for an unit sphere in four-dimensional space gives a contribution

$$
e^{-\mathcal{A}_{\mathrm{eff}}}=e^{\int_{0}^{S} d \lambda R / 6 \mu}=e^{\int_{0}^{S} d \lambda 1 / \mu}=e^{\kappa^{2} S}
$$

The path integral (2.31) had been solved in Refs. [5, 9] by

$$
K\left(\mathbf{p}_{b}, \mathbf{p}_{a} ; S\right)=\sum_{n=1}^{\infty} \frac{n^{2}}{2 \pi^{2}} P_{n}(\cos \vartheta) e^{-\left(\kappa^{2} n^{2}-\alpha^{2}\right) S / 2},
$$

where $P_{n}(\cos \vartheta)=\sin n \vartheta / n \sin \vartheta$ with $\vartheta$ being the angular between the four vectors $\hat{\mathbf{z}}_{b}$ and $\hat{\mathbf{z}}_{a}$ :

$$
\cos \vartheta=\hat{\mathbf{z}}_{b} \cdot \hat{\mathbf{z}}_{a}=\frac{\left(p_{b}^{2}-\kappa^{2}\right)\left(p_{a}^{2}-\kappa^{2}\right)+4 \kappa^{2} \mathbf{p}_{b} \cdot \mathbf{p}_{a}}{\left(p_{b}^{2}+\kappa^{2}\right)\left(p_{a}^{2}+\kappa^{2}\right)}
$$


We now complete the integration on $S$ in Eq. (2.30) and obtain the exact Green function of the Coulomb system in momentum space

$$
G\left(\mathbf{p}_{b}, \mathbf{p}_{a} ; E\right)=(2 \pi)^{3} \sum_{n=1}^{\infty} \frac{n^{2}}{2 \pi^{2}} P_{n}(\cos \vartheta) \frac{2}{2 E n^{2}+\alpha^{2}}
$$

We see that the poles display the correct energy levels of hydrogen spectra

$$
E_{n}=-\frac{\alpha^{2}}{2 n^{2}}, \quad n=1,2,3, \ldots
$$

This path integral derivation was first given by Kleinert in [8].

Here is the place that we can go over to the A-B-C system. Let us define the new quantum number $n_{r}=n-1$ and rewrite $(2.36)$ as

$$
G\left(\mathbf{p}_{b}, \mathbf{p}_{a} ; E\right)=(2 \pi)^{3} \sum_{n_{r}=0}^{\infty} \frac{\left(n_{r}+1\right)}{2 \pi^{2}} \frac{2}{2 E\left(n_{r}+1\right)^{2}+\alpha^{2}} C_{l}^{1}(\cos \vartheta)
$$

where $C_{l}^{1}(\cos \vartheta)=\sin \left(n_{r}+1\right) \vartheta / \sin \vartheta$ are the Gegenbauer polynomials [12, p. 218]. By defining the new variables

$$
u=\frac{\left(p_{b}^{2}-\kappa^{2}\right)}{\left(p_{b}^{2}+\kappa^{2}\right)}, \quad v=\frac{\left(p_{a}^{2}-\kappa^{2}\right)}{\left(p_{a}^{2}+\kappa^{2}\right)}
$$

with the addition theorem of the Gegenbauer polynomials [12, p. 223], $C_{l}^{1}(\cos \vartheta)$ has the expansion

$$
\begin{aligned}
C_{n_{r}}^{1}(\cos \vartheta)= & C_{n_{r}}^{1}\left(u \cdot v+\left(1-u^{2}\right)^{1 / 2}\left(1-v^{2}\right)^{1 / 2} \cos \Delta \vartheta\right) \\
= & \sum_{l=0}^{n_{r}} \frac{4^{l}(2 l+1) \Gamma\left(n_{r}-l+1\right) \Gamma^{2}(l+1)}{\Gamma\left(n_{r}+l+2\right)} \\
& \times\left(1-u^{2}\right)^{l / 2}\left(1-v^{2}\right)^{l / 2} C_{n_{r}-l}^{l+1}(u) C_{n_{r}-l}^{l+1}(v) P_{l}(\cos \Delta \vartheta),
\end{aligned}
$$

where $\Delta \vartheta$ is the angular between the unit vectors $\hat{\mathbf{p}}_{b}, \hat{\mathbf{p}}_{a}$ in the momentum space. Since the Legendre function has the expansion into the spherical harmonic

$$
P_{l}(\cos \Delta \vartheta)=\frac{4 \pi}{(2 l+1)} \sum_{k=-l}^{l} Y_{l k}\left(\hat{\mathbf{p}}_{b}\right) Y_{l k}^{*}\left(\hat{\mathbf{p}}_{a}\right)
$$

the exact Green's function becomes

$$
\begin{aligned}
G\left(\mathbf{p}_{b}, \mathbf{p}_{a} ; E\right)= & (2 \pi)^{3} \sum_{n_{r}=0}^{\infty} \sum_{l=0}^{n_{r}} \sum_{k=-l}^{l} \frac{\left(n_{r}+1\right)}{2 \pi^{2}} \frac{2}{2 E\left(n_{r}+1\right)^{2}+\alpha^{2}} \\
& \times \frac{4^{l}(2 l+1) \Gamma\left(n_{r}-l+1\right) \Gamma(l+k+1) \Gamma(l-k+1)}{\Gamma\left(n_{r}+l+2\right)} \\
& \times\left(1-u^{2}\right)^{l / 2}\left(1-v^{2}\right)^{l / 2} C_{n_{r}-l}^{l+1}(u) C_{n_{r}-l}^{l+1}(v) P_{l-k}^{(k, k)}\left(\cos \Theta_{b}\right) P_{l-k}^{(k, k)}\left(\cos \Theta_{a}\right) \\
& \times\left(\cos \Theta_{b} / 2 \cos \Theta_{a} / 2 \sin \Theta_{b} / 2 \sin \Theta_{a} / 2\right)^{k} \cdot e^{i k\left(\Phi_{b}-\Phi_{a}\right)} .
\end{aligned}
$$


Here, $(\Theta, \Phi)$ are the angular of the polar coordinates of the total momentum vector in momentum space, and the relation between the associated Legendre polynomial $P_{l}^{k}(x)$ and the Jacobi function $P_{n}^{(k, k)}(x)[10]$

$$
P_{l}^{k}(\cos \Theta)=(-1)^{k} \frac{\Gamma(1+k+l)}{\Gamma(1+l)}(\cos \Theta / 2 \sin \Theta / 2)^{k} P_{l-k}^{(k, k)}(\cos \Theta),
$$

has been used for obtaining Eq. (2.42) via

$$
Y_{l k}(\hat{\mathbf{p}})=Y_{l k}(\Theta, \Phi)=(-1)^{k}\left[\frac{2 l+1}{4 \pi} \frac{(l-k) !}{(l+k) !}\right]^{1 / 2} P_{l}^{k}(\cos \Theta) e^{i k \Phi}
$$

To proceed, we change summation indices by defining $n_{r}-l=n$, and a further change of the index of summation replaces $l$ with $q$ by defining $l-k=q$. We obtain from (2.42)

$$
\begin{aligned}
G\left(\mathbf{p}_{b}, \mathbf{p}_{a} ; E\right)= & (2 \pi)^{3} \sum_{n=0}^{\infty} \sum_{q=0}^{\infty} \sum_{k=-\infty}^{\infty} \frac{(n+q+k+1)}{2 \pi^{2}} \frac{2}{2 E(n+q+k+1)^{2}+\alpha^{2}} \\
& \times \frac{4^{q+k}[2(q+k)+1] \Gamma(n+1) \Gamma(q+1) \Gamma(q+2 k+1)}{\Gamma(n+2(q+k)+2)} \\
& \times\left(1-u^{2}\right)^{(q+k) / 2}\left(1-v^{2}\right)^{(q+k) / 2} C_{n}^{q+k+1}(u) C_{n}^{q+k+1}(v) P_{q}^{(k, k)}\left(\cos \Theta_{b}\right) P_{q}^{(k, k)}\left(\cos \Theta_{a}\right) \\
& \times\left(\cos \Theta_{b} / 2 \cos \Theta_{a} / 2 \sin \Theta_{b} / 2 \sin \Theta_{a} / 2\right)^{k} \cdot e^{i k\left(\Phi_{b}-\Phi_{a}\right)} .
\end{aligned}
$$

We are now prepared to include the influence of the A-B potential in (2.17). With the help of Poisson's summation formula [5, p. 124],

$$
\sum_{k=-\infty}^{\infty} f(k)=\int_{-\infty}^{\infty} d y \sum_{n=-\infty}^{\infty} e^{2 \pi n y i} f(y)
$$

we obtain for the Green function of the A-B-C system in momentum space

$$
\begin{aligned}
G\left(\mathbf{p}_{b}, \mathbf{p}_{a} ; E\right)= & (2 \pi)^{3} \sum_{n=0}^{\infty} \sum_{q=0}^{\infty} \sum_{k=-\infty}^{\infty} \frac{2}{2 \pi^{2}} \frac{2}{2 E\left(n+q+\left|k+\mu_{0}\right|+1\right)^{2}+\alpha^{2}} \\
& \times \frac{4^{\left(q+\left|k+\mu_{0}\right|\right)}\left[2\left(q+\left|k+\mu_{0}\right|\right)+1\right] \Gamma(n+1) \Gamma(q+1) \Gamma\left(q+2\left|k+\mu_{0}\right|+1\right)}{\Gamma\left(n+2\left(q+\left|k+\mu_{0}\right|\right)+2\right)} \\
& \times\left(\frac{2 \kappa p_{b}}{p_{b}^{2}+\kappa^{2}}\right)^{q+\left|k+\mu_{0}\right|} \cdot\left(\frac{2 \kappa p_{a}}{p_{a}^{2}+\kappa^{2}}\right)^{q+\left|k+\mu_{0}\right|} \\
& \times C_{n}^{q+\left|k+\mu_{0}\right|+1}\left(\frac{p_{b}^{2}-\kappa^{2}}{p_{b}^{2}+\kappa^{2}}\right) \cdot C_{n}^{q+\left|k+\mu_{0}\right|+1}\left(\frac{p_{a}^{2}-\kappa^{2}}{p_{a}^{2}+\kappa^{2}}\right) \\
& \times P_{q}^{\left(\left|k+\mu_{0}\right|,\left|k+\mu_{0}\right|\right)}\left(\cos \Theta_{b}\right) \cdot P_{q}^{\left(\left|k+\mu_{0}\right|,\left|k+\mu_{0}\right|\right)}\left(\cos \Theta_{a}\right) \\
& \times\left(\cos \Theta_{b} / 2 \cos \Theta_{a} / 2 \sin \Theta_{b} / 2 \sin \Theta_{a} / 2\right)^{\left|k+\mu_{0}\right|} \cdot e^{i k\left(\Phi_{b}-\Phi_{a}\right)} .
\end{aligned}
$$


We see that the energy spectra are determined by the poles

$$
E_{n, q, k}=-\frac{\alpha^{2}}{2\left(n+q+\left|k+\mu_{0}\right|+1\right)^{2}} .
$$

This agrees with the result in Refs. [10,11]. The wave functions can be read off by giving the form of the spectral representation

$$
\begin{aligned}
G\left(\mathbf{p}_{b}, \mathbf{p}_{a} ; E\right)= & \sum_{n=0}^{\infty} \sum_{q=0}^{\infty} \sum_{k=-\infty}^{\infty}\left[\frac{\pi^{3}\left(p_{b}^{2}+\kappa^{2}\right)^{2}\left(p_{a}^{2}+\kappa^{2}\right)^{2}}{2 \kappa^{5}\left(n+q+\left|k+\mu_{0}\right|+1\right)^{2}}\right] \frac{1}{E-E_{n, q, k}} \\
& \times \frac{2^{3} \kappa^{5}\left(n+q+\left|k+\mu_{0}\right|+1\right)}{\pi^{2}} \cdot 4^{\left(q+\left|k+\mu_{0}\right|\right)} \\
& \times \frac{\left[2\left(q+\left|k+\mu_{0}\right|\right)+1\right] \Gamma(n+1) \Gamma(q+1) \Gamma\left(q+2\left|k+\mu_{0}\right|+1\right)}{\Gamma\left(n+2\left(q+\left|k+\mu_{0}\right|\right)+2\right)} \\
& \times \frac{1}{\left(p_{b}^{2}+\kappa^{2}\right)^{2}} \cdot \frac{1}{\left(p_{a}^{2}+\kappa^{2}\right)^{2}} \cdot\left(\frac{2 \kappa p_{b}}{p_{b}^{2}+\kappa^{2}}\right)^{q+\left|k+\mu_{0}\right|} \cdot\left(\frac{2 \kappa p_{a}}{p_{a}^{2}+\kappa^{2}}\right)^{q+\left|k+\mu_{0}\right|} \\
& \times C_{n}^{q+\left|k+\mu_{0}\right|+1}\left(\frac{p_{b}^{2}-\kappa^{2}}{p_{b}^{2}+\kappa^{2}}\right) \cdot C_{n}^{q+\left|k+\mu_{0}\right|+1}\left(\frac{p_{a}^{2}-\kappa^{2}}{p_{a}^{2}+\kappa^{2}}\right) \\
& \times P_{q}^{\left(\left|k+\mu_{0}\right|,\left|k+\mu_{0}\right|\right)}\left(\cos \Theta_{b}\right) \cdot P_{q}^{\left(\left|k+\mu_{0}\right|,\left|k+\mu_{0}\right|\right)}\left(\cos \Theta_{a}\right) \\
& \times\left(\cos \Theta_{b} / 2 \cos \Theta_{a} / 2 \sin \Theta_{b} / 2 \sin \Theta_{a} / 2\right)^{\left|k+\mu_{0}\right|} \cdot e^{i k\left(\Phi_{b}-\Phi_{a}\right)}, \\
= & \sum_{n=0}^{\infty} \sum_{q=0}^{\infty} \sum_{k=-\infty}^{\infty}\left[\frac{\pi^{3}\left(p_{b}^{2}+\kappa^{2}\right)^{2}\left(p_{a}^{2}+\kappa^{2}\right)^{2}}{2 \kappa^{5}\left(n+q+\left|k+\mu_{0}\right|+1\right)^{2}}\right] \frac{1}{E-E_{n, q, k}} \Psi_{n, q, k}\left(\mathbf{p}_{b}\right) \Psi_{n, q, k}^{*}\left(\mathbf{p}_{a}\right)+\cdots
\end{aligned}
$$

From this we identify the wave functions as

$$
\begin{aligned}
\Psi_{n, q, k}(\mathbf{p})= & \frac{2 \sqrt{2 \kappa^{5}}}{\pi} 2^{\left(q+\left|k+\mu_{0}\right|\right)}\left(n+q+\left|k+\mu_{0}\right|+1\right)^{1 / 2} \\
& \times\left(\frac{\left[2\left(q+\left|k+\mu_{0}\right|\right)+1\right] \Gamma(n+1) \Gamma(q+1) \Gamma\left(q+2\left|k+\mu_{0}\right|+1\right)}{\Gamma\left(n+2\left(q+\left|k+\mu_{0}\right|\right)+2\right)}\right)^{1 / 2} \\
& \times \frac{1}{\left(p^{2}+\kappa^{2}\right)^{2}} \cdot\left(\frac{2 \kappa p}{p^{2}+\kappa^{2}}\right)^{q+\left|k+\mu_{0}\right|} \cdot C_{n}^{q+\left|k+\mu_{0}\right|+1}\left(\frac{p^{2}-\kappa^{2}}{p^{2}+\kappa^{2}}\right) \\
& \times P_{q}^{\left(\left|k+\mu_{0}\right|,\left|k+\mu_{0}\right|\right)}(\cos \Theta) \cdot(\cos \Theta / 2 \sin \Theta / 2)^{\left|k+\mu_{0}\right|} \cdot e^{i k \Phi} .
\end{aligned}
$$

The normalization condition

$$
\int d^{3} p \Psi_{n, q, k}(\mathbf{p}) \Psi_{n, q, k}^{*}(\mathbf{p})=1
$$

can be easily checked by using the recursion formula

$$
z C_{n}^{v}(z)=\frac{1}{2(n+v)}\left[(n+1) C_{n+1}^{v}(z)+(n+2 v-1) C_{n-1}^{v}(z)\right]
$$


and the following orthogonality relations of the Jacobi function [5, p. 383]

$$
\int_{-1}^{-1} d x(1-x)^{\alpha}(1+x)^{\beta} P_{n}^{(\alpha, \beta)}(x) P_{m}^{(\alpha, \beta)}(x)=\frac{2^{\alpha+\beta+1}}{\alpha+\beta+2 n+1} \frac{\Gamma(\alpha+n+1) \Gamma(\beta+n+1)}{n ! \Gamma(\alpha+\beta+n+1)} \delta_{m, n},
$$

as well as the Gegenbauer polynomials [5, p. 378],

$$
\int_{-1}^{1} d x C_{n}^{\lambda}(x) C_{m}^{\lambda}(x)\left(1-x^{2}\right)^{\lambda-1 / 2}=\frac{\pi 2^{1-2 \lambda} \Gamma(n+2 \lambda)}{n !(\lambda+n) \Gamma^{2}(\lambda)} \delta_{m, n} .
$$

It is of interest to evaluate the average values of the square of the momentum in the various quantum states:

$$
\left\langle P^{2}\right\rangle=\int_{0}^{\infty} \int_{0}^{\pi} \int_{0}^{2 \pi} P^{2} d P \sin \Theta d \Theta d \Phi \Psi_{n, q, k}(\mathbf{p}) P^{2} \Psi_{n, q, k}^{*}(\mathbf{p}) .
$$

With the help of Eqs. (2.53) and (2.54), we find

$$
\left\langle P^{2}\right\rangle=\kappa^{2}=\left(\frac{\alpha}{n+q+\left|k+\mu_{0}\right|+1}\right)^{2} .
$$

This quantity characterizes the modified circular Bohr orbit of the A-B-C system. For the hydrogen atom it specifics the square momentum of the electron in a circular Bohr orbit with the same total quantum number. Indeed, for $\mu_{0}=0$, when there is no A-B effect, Eq. (2.50) reduces to the wave functions of the pure Coulomb system

$$
\begin{aligned}
\Psi_{\tilde{n}, l, k}(\mathbf{p})= & \frac{2 \sqrt{2 \kappa^{5}}}{\pi} 2^{l} \Gamma(l+1) \sqrt{\frac{4 \pi \tilde{n} \Gamma(\tilde{n}-l)}{\Gamma(\tilde{n}+l+1)}} \\
& \times \frac{1}{\left(p^{2}+\kappa^{2}\right)^{2}} \cdot\left(\frac{2 \kappa p}{p^{2}+\kappa^{2}}\right)^{l} \cdot C_{\tilde{n}-l-1}^{l+1}\left(\frac{p^{2}-\kappa^{2}}{p^{2}+\kappa^{2}}\right) \cdot Y_{l k}(\Theta, \Phi),
\end{aligned}
$$

where we have defined $\tilde{n}=n+l+1(\tilde{n}=1,2,3, \ldots, l=0,1,2, \ldots)$. Wave functions were first obtained by Podolanski and Pauling by carrying out the Fourier transformation on the wave function of the position space in Ref. [13]. From the Schrödinger equation in the momentum space, the Coulomb system was solved by Fock $[14,15]$. The average values of the square are given by

$$
\left\langle P^{2}\right\rangle=\kappa^{2}=\left(\frac{\alpha}{\tilde{n}}\right)^{2} .
$$

This special case of (2.56) is the same as for a circular Bohr orbit, as discussed in the classical paper of Pauling [13].

\section{CONCLUSION}

We have derived the exact Green function of the A-B-C system in momentum space from path integrals. From this, the wave functions as well as the energy spectra have been extracted. Since the 
dynamics of the system in momentum space is on a curved manifold, our discussion offers another piece of evidence in answering the historic question of different Hamiltonians in curved spaces.

\section{ACKNOWLEDGMENTS}

I thank the referee for his critical reading, marks, and comment. The author has benefited from discussion with Dr. P. G. Luan.

\section{REFERENCES}

1. R. P. Feynman, Ph.D. thesis, Princeton University, May 1942.

2. R. P. Feynman, Rev. Mod. Phys. 20 (1948), 367.

3. J. Schwinger, Phys. Today 43 (1989), 42.

4. M. Gell-mann, Phys. Today 43 (1989), 48.

5. H. Kleinert, "Path Integrals in Quantum Mechanics, Statistics and Polymer Physics," World Scientific, Singapore, 1995.

6. C. Grosche and F. Steiner, "Handbook of Feynmann Path Integrals," Springer Tracts in Modern Physics, Vol. 145, Springer-Verlag, Berlin, 1998.

7. B. S. DeWitt, Rev. Mod. Phys. 29 (1957), 337.

8. H. Kleinert, Phys. Lett. A 252 (1999), 277.

9. D. H. Lin, J. Phys. A 30 (1997), 3201.

10. D. H. Lin, J. Math. Phys. 41 (2000), 2723.

11. D. H. Lin, J. Math. Phys. 40 (1999), 1246.

12. W. Magnus, F. Oberhettinger, and R. P. Soni, "Formulas and Theorems of the Special Function of Mathematical Physics," Springer-Verlag, Berlin, 1966.

13. B. Podolanski and L. Pauling, Phys. Rev. 34 (1929), 109.

14. V. Fock, Z. Phys. 29 (1935), 145.

15. H. A. Bethe and E. E. Salpeter, "Quantum Mechanics of One- and Two-Electron Atoms," Plenum, New York, 1977. 